\title{
Self-assembling DNA Nanostructures for Patterned Molecular Assembly
}

Thomas H. LaBean ${ }^{a}$, Kurt V. Gothelf ${ }^{b}$, and John H Reif ${ }^{\mathfrak{c}}$

${ }^{a}$ Departments of Computer Science and Chemistry, Duke University, Durham, North Carolina

27708 USA.Email: thomas.labean@duke.edu; Tel: (919)660-1565

${ }^{b}$ Department of Chemistry, Aarhus University, Langelandsgade 140, 8000 Aarhus C Denmark.

Email:kvg@chem.au.dk; Tel: (+45)89423907

${ }^{c}$ Department of Computer Science, Duke University, Durham, North Carolina 27708 USA.

Email: reif@cs.duke.edu; Tel: (919)660-65685

\begin{abstract}
The Chapter describes the use of DNA for molecular-scale self-assembly. DNA-nanostructures provide a versatile toolbox with which to organize nanoscale materials. We begin with a discussion of DNA-nanostructures, starting with the self-assembly of various building-blocks known as DNA tiles. We describe how these can be made to self-assemble into two and threedimensional lattices. We discuss various methods for the programmed assembly of patterned and/or shaped two and three-dimensional DNA-nanostructures, including their use to produce beautiful algorithmic assemblies displaying fractal design patterns. The resulting large DNAnanostructures provide multiple attachment sites within and between tiles for complex programmed structures and lead to diverse possibilities for scaffolding useful constructs and templating interesting chemistries. We describe methods for assembly of various biomolecules and metallic nanoparticles onto DNA-nanostructures, and also the assembly of various materials using DNA-nanostructures. Finally, we conclude the chapter with a discussion of various challenges.
\end{abstract}




\section{Introduction}

Self-assembly is one of the key approaches that may enable future methods for building nanostructures and nanodevices. ${ }^{1}$ Our current ability to form nanostructures by self-assembly is, however, quite limited compared to the power of lithographic techniques for the formation of solid structures in bulk material and in particular, electronic circuits at the nanoscale. Lithography is used to form the most complex human-constructed objects: microprocessors, which have been manufactured with upwards of billions of precisely patterned elements. However, top-down techniques such as lithography are limited in scale, where as bottom-up methods of self-assembly are not.

The motivation and inspiration to exploring self-assembly can literally be found within ourselves, since living organisms such ourselves are the most advanced nanosystems known, far exceeding the complexity of human-engineered nanostructures. The fantastically complex machinery of living organisms is composed primarily of organic molecules and polymers. It is formed by and operates by self-assembly. The precision and efficiency of the self-assembly process in cells derives from specific molecular interactions between proteins, DNA and RNA in particular, and other compounds including lipids, carbohydrates, and small molecules. The question is: can we use techniques inspired from the cell's self-assembly machinery to assemble artificial nanostructures? Also, what materials should we use as building blocks to direct the self-assembly process at the molecule scale?

Proteins, antibodies, peptides and their small molecule affinity substrates are efficient and highly specific in self-assembly processes and if only few types of specific interactions are required they might be the best choice. The importance of their role in molecular biology, medicine and nanoscience cannot be underestimated. However, when it comes to the individual encoding of multiple building blocks for the assembly into complex nanostructures, their use may be limited due to their diversity in structure and differences in the nature of their self-interactions or size. These materials have their own, extensive technical literature, which will not be discussed here. 
In contrast, DNA nanostructures have an easily predictable secondary structure due to the well the understood properties of single stranded DNA base-pairing and the double helix structure of double stranded DNA as functions of their environment (temperature and buffer solution), ${ }^{2-4}$ allowing for software to be developed for computer aided design of the sequences composing DNA nanostructures. ${ }^{5,6}$ There is also a well-established biotechnology for constructing DNA sequences and for executing operations on DNA sequences. There are known techniques for attaching other molecules to specific locations on DNA sequences. Hence DNA appears to be an ideal material for achieving complex self-assembly, and we will center our attention on this topic.

\section{Overview of DNA-Nanostructures}

The last few years have seen a great number of advances in our ability to construct complex nanostructures from nucleic acid building materials. ${ }^{7-9}$ The study of artificial DNA structures for applications in nanotechnology began in the early 80's when Seeman sought to design and construct periodic matter and discrete objects assembled from synthetic DNA oligonucleotides. ${ }^{10}$ He noted that simple double-helical DNA could only be used for the construction of linear assemblies and that more complex building blocks would be required for 2- and 3-dimensional constructs. He also noted that biological systems make use of branched base-pairing complexes such as forks (3-arm junctions) found in replicating DNA and Holliday intermediates (4-arm junctions) found in homologous recombination complexes. These natural branch junction motifs exposed a potential path toward multi-valent structural units. A Holliday junction is formed by four strands of DNA (two identical pairs of complementary strands) where double-helical domains meet at a branch point and exchange base-pairing partner strands. The branch junctions in recombination complexes are free to diffuse up and down the paired homologous dsDNA domains since the partners share sequence identity all along their lengths. Seeman showed that by specifically designing sequences which were able to exchange strands at a single specified point and by breaking the sequence symmetry which allowed the branch junction to migrate, ${ }^{11}$ immobile junctions could be constructed and used in the formation of stable and rigid DNA building blocks. These building blocks (known as DNA tiles), especially double-crossover (DX) complexes ${ }^{12}$ became the initial building blocks for construction of periodic assemblies and the 
formation of the first uniform 2-dimensional crystals of DNA known as DNA lattices. ${ }^{13}$ They also can be used to form long tubes. ${ }^{14}$

\section{Insert Fig. 1}

A large number of distinct DNA tile types have now been designed and prototyped; some examples are shown in Fig. 1. The high thermal stability ( $\mathrm{T}_{\mathrm{m}}$ up to at least $70^{\circ} \mathrm{C}$ ) of some DNA tiles, the ability to program tile-to-tile association rules via ssDNA sticky-ends, and the wide range of available attachment chemistries make these structures extremely useful as molecular scale building blocks for diverse nanofabrication tasks. The process of DNA nanostructure and sequence design was laid out very effectively in a recent review article. ${ }^{15}$ DNA tiles produced to date have contained double-helical DNA domains as structural members and branch junctions crossovers as connectors. The use of paired crossovers greatly increases the stiffness of the tiles over that of linear dsDNA. Following the success of the DX lattices, triple-crossover (TX) tiles and their 2D uniform lattices and tubes were demonstrated. ${ }^{16-20}$ Recently, DDX tiles containing four double helices and four crossover points have also been demonstrated. ${ }^{21}$

Since DX, DDX and TX tiles are designed with their helices parallel and coplanar their lattices tend to grow very well in the dimension parallel with the helix axes and fairly poorly in the dimension perpendicular to it. Elimination of this problem and growth of lattices with a square aspect ratio was the primary motivation behind the design of the cross-tile, ${ }^{22}$ which allows for a uniform growth of two dimensional lattices in both these directions. Long DNA nanotubes (of up to $15 \mu \mathrm{m}$ length) and large 2D lattices (extending many square micrometers) have been assembled from cross-tiles (Fig. 2). The design of the 2D cross-tile lattices made use of an interesting corrugation method ${ }^{22}$ for providing symmetry in both the horizontal and vertical directions, by the use of a rotations and flips of neighboring the cross-tiles so as to cancel deformations that otherwise would limit lattice growth. Recently a variant of the cross-tile ${ }^{23}$ was developed that provides an improved symmetry in both the horizontal and vertical directions, resulting in even larger 2D lattices (extending to millimeters). 


\section{Insert Fig. 2}

A variety of other tile shapes have been prototyped beyond the rectangular and square tiles shown above. Lattice with rhombus-like units have been made in which the helix crossing angles are closer to the relaxed $\sim 60^{\circ}$ angles observed in biological Holliday junctions. ${ }^{24}$ At least three different versions of triangular DNA tiles have been prototyped (Fig. 3), one which tiles the plane with triangles ${ }^{25}$ and two types which form hexagonal patterns. ${ }^{26,27}$ Such triangular lattices have not been show to grow as large as those from rectangular and square tiles, but they may be useful for assembly applications where slightly more structural flexibility is desired. They have also demonstrated some interesting multilayer structures with symmetrical stacking interactions. ${ }^{27}$

Insert Fig. 3

DNA tiles have also been assembled using paranemic interactions between pairs of parallel helices. $^{28}$

\section{D DNA Nanostructures}

Three-dimensional building blocks and periodic matter constructed of DNA have been long-term goals of this field. In addition to forming 2D lattices and tubes, DNA tiles can be used to form 3D lattices. If guest molecules can be incorporated into 3D DNA lattices, applications include:

- The assembly of 3D molecular electronics circuits and memory, and

- The molecular scaffold for structure determination of guest molecules via x-ray crystallography studies.

Non-planar tiles represent one strategy for expanding the tiling into the third dimension. DNA tiles which hold their helical domains in non-planar arrangements have been designed, for example a three-helix bundle (Fig. 4). ${ }^{29}$ and a six-helix bundle. ${ }^{30}$, although initial attempts at 3D 
DNA nanostructures using these particular tiles have not yet succeeded. Recently Seeman ${ }^{31}$ has demonstrated a 3D DNA hexagonal lattice formed from a single 13 base DNA sequence that formed stacked layers of parallel helices with base-pairing between adjacent layers. However, these 3D DNA lattices are not yet regular enough to allow for the application to structure determination of guest molecules via x-ray crystallography.

\section{Insert Fig. 4}

Another approach is to form 3D polyhedral DNA nanostructures that can then assembly to regular lattices. Early attempts to build a cube ${ }^{32}$ and a truncated octahedron ${ }^{33}$ with dsDNA edges and branch junction vertices met with some success, but the final constructs were produced in very low yields. More recently, a tetrahedral unit with short double helical edges was constructed in much higher yield. ${ }^{34}$ Perhaps the most impressive experimental success yet in DNA-based 3D nanostructures produced an octahedron with DX-like edges. ${ }^{35}$ This study was noteworthy in that the 1.7 kilobase DNA strand (which folded with the help of five short oligonucleotides) was produced as a single piece by PCR-based assembly, and the octahedron was formed in sufficient yield to permit structural characterization by cryo-electron microscopy.

\section{Programmed Patterning of DNA-Nanostructures}

Besides 2D and 3D periodic lattices, another long-term goal of DNA self-assembly studies has been the generation of complex, non-periodic patterns on lattices. There are at least three techniques for doing this:

(1) The first is the use of algorithmic self-assembly, whereby patterns are formed using a small tile set whose sticky-ends represent tile association rules that promote lattice formation according to the specific rules of the encoded algorithm. The first demonstrations of algorithmic self-assembly used DNA tiles to demonstrate the execution of various Boolean and arithmetic operations at the molecular scale; the computations occurred during the assembly of linear sequences of DNA tiles that preferentially bound to each other according to the computational rules. ${ }^{17,36}$ Subsequently, 2D demonstrations of algorithmic self-assembly method using DNA tiles have provided some of the most complex patterns yet demonstrated via molecular self-assembly; 
an example is seen in the Sierpinski triangle pattern shown in Fig. $5 .{ }^{37}$ In principle, any arbitrary structure which can be specified by a set of encoded association rules can be expected to form via algorithmic self-assembly, ${ }^{38}$ albeit at some yield $<100 \%$ and with some error rate $>0 \%$. Various schemes have been developed to reduce errors in algorithmic self-assembly but with the sideeffect of increasing the size of the lattice as well as the number of tile types. ${ }^{39-41}$ Compact errorresilient designs were later developed which provide a reduction of assembly errors without increase in the size of the computational lattice. ${ }^{42,43}$

\section{Insert Fig. 5}

(2) Another method for programmed patterning of DNA-nanostructures is the use of stepwise or hierarchal self-assembly, whereby patterns are formed in multiple stages, incorporating as subcomponents patterned nanostructures formed in prior stages. A recent demonstration of this technique provided the molecular-scale self-assembly of fixed-size DNA lattices patterned in any arbitrary way (see Fig. 6). ${ }^{44}$ This study showed that by minimizing the number of sequential steps in the assembly process, the overall yield of target structure would be maximized.

(3) A final and very promising method is the use of directed self-assembly, whereby patterns are formed via the use of molecules that control in some way the self-assembly process so as to form the intended pattern. Directed self-assembly of 1D patterned DNA lattices was first demonstrated by the use of scaffold strands to provide the specification of inputs to the computational assemblies mentioned above. ${ }^{36,38}$ Directed self-assembly of 2D patterned DNA lattices was then demonstrated by the use of scaffold strands that were incorporated into each of the rows of a 2D DNA lattice, allowing for the display of binary sequences as a 2D barcode patterns that can be viewed by AFM imaging. ${ }^{45}$ Rothemund made the most impressive use of directed self-assembly to date, using a 7-kilobase scaffold strand which folded into arbitrary 2D shapes and patterns with the help of multiple short oligonucleotides that specify the shape and patterning (see Fig. 6). ${ }^{46}$

Finite-sized arrays were also reported in another DNA system and in RNA. ${ }^{47,48}$ Previous DNA tiling systems all resulted in unbounded growth of lattice and consequently polydisperse products 
following annealing. These demonstrations of finite-sized arrays represent another step toward increased control of self-assembled molecular systems.

Insert Fig. 6

The 2D lattices and 3D structures assembled from DNA and described in this and the prior sections represent interesting objects in their own right, but their real usefulness will come from their application as scaffolds and templates upon which chemistry is performed or with which heteromaterials are organized into functioning nanodevices. We will use the term $D N A$ programmed patterned assembly to denote this use of DNA lattices to organize heteromaterials. We will return to some of these applications later in this chapter.

\section{DNA-Programmed Assembly of Biomolecules}

Assembly of other biomolecules on DNA templates and arrays may prove useful for fabrication of biomimetics and other devices with applications such as biochips, immunoassays, biosensors, and a variety of nanopatterned materials. The logical end to the shrinking of microarrays is the self-assembled DNA nanoarray with a library of ligands distributed at addressable locations to bring analyte detection down to the single molecule level. We will return to complex DNA tiling structures momentarily, but first we will look at simpler dsDNA systems.

The conjugation of DNA and streptavidin via a covalent linker was reported by Niemeyer et al. in 1994, and these conjugates were applied for DNA-programmed assembly on a macroscopic DNA array on a surface and in a nanoscale array made by aligning DNA-tagged proteins to specific

positions along a oligonucleotide template. ${ }^{49-51}$ The covalent attachment of an oligonucleotide to streptavidin provides a specific recognition domain for a complementary nucleic acid sequence. In addition the binding capacity for four biotin molecules is utilized as biomolecular adapters for positioning biotinylated components along a nucleic acid backbone. 
Besides duplex DNA structures, more complex self-assembling DNA tiling structures have been used to organize biomolecules into specific spatial patterns. DNA nanostructures covalently labeled with ligands have been shown to bind protein molecules in programmed patterns, for example, making use of the popular biotin/avidin pair, arrays of evenly spaced streptavidin molecules were assembled on DNA tile lattice. ${ }^{22}$ On cross-tile lattice, individual streptavidin molecules are visible as separate peaks in the AFM image (Fig. 7). Single molecule detection could be achieved on DNA nanoarrays displaying a variety of protein binding ligands.

\section{Insert Fig. 7}

Further design evolution of the cross-tile system to a two tile type (A and B) tile set allowed for somewhat more complex structures and patterns. ${ }^{52}$ In this study, some size control of lattice and partial addressability were demonstrated, but the display patterns were still periodic and symmetric (Fig. 8). In ongoing experiments, finite-sized objects with independent addressing have been used to assemble a range of specifically patterned protein arrays in high yield. ${ }^{44}$

\section{Insert Fig. 8}

Another exciting future use for biomolecules specifically patterned on self-assembled DNA nanostructures is the specific deposition of inorganic materials via crystal nucleation. Natural peptides and proteins have been implicated in the growth of nano-patterned silica by living

organisms. ${ }^{53}$ Peptides and RNA sequences have been artificially evolved by in vitro selection to specifically bind and precipitate or crystallize various semi-conductors and metals. ${ }^{54,55}$ Patterning these species on 3D DNA lattices could provide a method for bottom-up assembly and controlled deposition resulting in a wide variety of complex inorganic structures for use in nanoelectronics, photonics, and other fields.

\section{DNA-programmed assembly of materials}


In analogy to the immobilization of DNA on a variety of solid surfaces, the conjugation of DNA and analogs with metal nanoparticles, semiconductor nanoparticles and polymer particles is becoming increasingly important. In particular DNA-directed assembly of gold nanoparticles has been studied in much detail, primarily due to the stability of and ease of preparation of gold nanoparticle conjugates and due to the interparticle distance-dependent plasmon resonance absorption. Mirkin et al. and Alivisatos et al. reported pioneering work on the assembly of gold nanoparticles by hybridization of DNA-nanoparticle conjugates in $1996 .^{56,57}$ Since then such DNA-conjugated materials have found several applications e.g. for biosensors and the reader is addressed to recent excellent reviews in this book and elseware ${ }^{58-61}$ for a detailed overview. Here the main focus will be on the assembly of materials in DNA-lattices on surfaces. Combining DNA's ability to organize nanomaterials with the diverse and programmable structures available from self-assembling DNA tile lattice strategies has resulted in several initial steps toward the bottom-up assembly of nanomaterials that may prove useful as electronic components. A TX tile assembly was used to align a modest number of $5 \mathrm{~nm}$ gold particles in single and double layer queues. ${ }^{17}$ This construction used tiles containing integral biotin-labeled DNA strands and streptavidin bound gold particles. In another study, DX tile arrays were used to pattern $6 \mathrm{~nm}$ gold particles into precisely spaced rows covering micrometer scale areas as shown in Fig. 9. ${ }^{62}$ This study featured gold nanoparticles labeled with $\mathrm{T}_{15}$ oligonucleotides which base-paired with assembled DX lattice displaying single-strand $A_{15}$ sequences hanging off certain tiles. A pair of recent papers from the Yan group have demonstrated useful improvements to this method. ${ }^{63,64}$ The ability to organize electrically active species such as gold using DNA points the way toward the templating of complex devices and circuits for applications in nanoelectronics.

\section{Insert Fig. 9}

DNA-programmed assembly of materials other than gold nanoparticles has also been reported. Such materials include, semiconductor nanoparticles, ${ }^{65,66}$ nanorods, ${ }^{67}$ mesoscale particles, ${ }^{68,69}$ and dendrimers ${ }^{70,71}$ Most of these examples are based on linear assembly of two complementary DNA strands leading to dimers or aggregates. Many examples of nanowires templated on DNA 
molecules by a variety of electroless deposition protocols (including fabrication of a field effect transistor $^{72}$ ) have also been reported, but these are beyond the scope of this article.

Carbon nanotubes are one of the most promising materials for nanoscience due to their unique structure and mechanic and electronic properties. ${ }^{73}$ In recent years chemical conjugation of organic and bioorganic compounds with carbon nanotubes is a field that has developed rapidly. ${ }^{74}$ The ability to control the exact positioning of multiple carbon nanotubes by means of DNAprogrammed assembly would be a major achievement in nanoscience. In a few reports, the conjugation of carbon nanotubes with $\mathrm{DNA}^{75,76}$ and with PNA has been described. ${ }^{77}$ In these reports carbon nanotubes were shortened into fragments by oxidation, resulting in carbon nanotubes fragments with carboxyl groups in the terminal positions and to some extend in their sites walls. Covalent coupling of 5'-amino DNA-sequences or PNA to carboxyl groups at the nanotubes led to the formation of carbon nanotubes coupled with DNA or PNA sequences. Carbon nanotubes containing 12-mer PNA-sequences were annealed with dsDNA sequences containing 12-mer sticky ends and imaged by $\mathrm{AFM}^{77}$ In the work by Dai et al., multi-wall carbon nanotubes (MWNTs) and single-wall carbon nanotubes (SWNT) functionalized with 20-mer DNA sequences were annealed with complementary sequences attached to gold nanoparticles. ${ }^{76}$ The resulting aggregates were deposited on mica and imaged by AFM. The images revealed the occasional interconnection of individual MWNTs by a gold nanoparticle. In a recent report by Chen et al. the self-assembly of DNA functionalized single-walled carbon nanotubes was studied and atomic force microscopy images showed that highly branched structures were formed for the DNA functionalized SWNTs. ${ }^{78}$

In another study, SWNT were assembled between prepositioned metal electrodes via complementary DNA base-pairing by ssDNA on the gold electrodes (thiol-labeled oligos) and the oxidized SWNTs (3'-amino-labeled oligos) as shown in Fig. $10{ }^{79}$ Electrical conductivity between the electrode pairs was shown to be highly dependent on the presence of complementary DNA on the electrodes and nanotubes. These initial investigations of carbon nanotube-DNA conjugates hold great promise for future developments in assembly of nanotube structures with useful electronic and mechanical properties.

Insert Fig. 10 


\section{Laboratory Methods}

The common methods utilized in DNA-based molecular assembly have been described in detail in the papers (and their supporting documents) cited throughout this chapter. An overview of the most central methods - i) molecular assembly by thermal annealing and ii) examination of 2D nanostructures by AFM imaging - will be included here as brief summaries.

Annealing for DNA Assembly. Formation of specific hybridization complexes from designed DNA strands takes place by slow annealing. First, custom oligonucleotides are obtained from a commercial vendor and purified by polyacrylamide gel electrophoresis under denaturing conditions. ${ }^{16}$ Complexes are formed by mixing stoichiometric quantities of the required strands (as estimated by their absorbance at $260 \mathrm{~nm}$ ) in TAE/Mg buffer (20 mM Tris-acetate [pH 7.6], 2 mM EDTA, $12.5 \mathrm{mM} \mathrm{MgCl}_{2}$ ). The concentration of DNA is typically between 1.0 and $0.05 \mu \mathrm{M}$ (per strand) in a volume of $10-100 \mu \mathrm{L}$. Mixed oligonucleotide solutions are then cooled slowly from $90{ }^{\circ} \mathrm{C}$ to $20{ }^{\circ} \mathrm{C}$ to facilitate hybridization. This annealing step can be achieved by sealing the DNA solution in a microfuge tube and floating the tube in a liter or two of $95^{\circ} \mathrm{C}$ water in a covered styrofoam box. Alternatively, a heating block or thermal cycler can be programmed to step down from $95^{\circ} \mathrm{C}$ to room temperature over the course of the annealing step. Styrofoam box annealing chambers can take up to 48 hours to cool down while a heating block, turned off and left on the bench can cool in under an hour. The length of the annealing step depends somewhat on the complexity of the nanostructure to be formed, the number of component strands required, and the nature of the assembly process (single-step, hierarchical, etc.) with less time required for simpler structures.

AFM Imaging. The majority of nanostructures produced so far by DNA assembly have been 2D and have been examined by atomic force microscopy on either wet or dry samples. For imaging under buffer, a 3 - $5 \mu \mathrm{L}$ DNA sample is spotted on freshly cleaved mica and left to adsorb to the surface for around 3 minutes, then $30 \mu \mathrm{L}$ TAE/Mg buffer is placed onto the mica. Another $30 \mu \mathrm{L}$ of buffer is pipetted between the AFM tip and the tip-holder so that when the tip and sample come together no air will be trapped above the tip. Imaging under buffer requires a fluid cell for the AFM. For imaging under air a 3 - $5 \mu \mathrm{L}$ DNA sample is spotted on freshly cleaved mica and left to adsorb to the surface for around 3 minutes, then the sample is wicked off with filter paper, $30 \mu \mathrm{L}$ of distilled water is used to rinse the mica which is then dried under a gentle stream of 
nitrogen. It has been found that imaging under buffer provides higher resolution analysis, and that "tapping mode" imaging is typically preferred over "contact mode".

\section{Conclusions}

We are now learning the basics of how to form DNA nanostructures with moderately high complexity. Consider for the moment just 2D nanostructures patterned at the molecular scale by a binary array of pixels. Let us define the complexity of such a patterned nanostructures to be the total number of pixels on the array that can be set on or off via programmed assembly. The prior review of patterned self-assembly results indicates that the experimentally achieved results for 2D patterning using DNA self-assembly has rapidly increased in complexity over the last few years (and this rate of increase seems to be considerably exceeding the rate that lithographicallybased patterning has increased in complexity over the same period). Thus we are optimistic about the further improvements in complexity of patterning using DNA self-assembly in future years.

In the ultimate development of bottom-up nanofabrication strategies it may be possible to assemble large numbers of easily available building blocks using patterned DNA nanostructures to position a wide variety of materials. Depending on the nature and programming of the building blocks they will self-assemble into complex nanostructures with the required properties. Such

properties might include: enzyme-like properties, electronic circuits with efficient contacts to electrodes at larger length scales, memory storage devices, drug delivery robots, multifunctional diagnostic devices for in vivo application, or even systems capable of self-replication.

\section{Acknowledgements}

Supported by NSF Grants EIA-0218376, CCR-0326157, CCF-0523555 and CCF-0432038. 


\section{References}

[1] Whitesides, G.M. and Grzybowski, B. (2002) Self-assembly at all scales. Science 295, 24182421.

[2] SantaLucia, J. and Hicks, D. (2004) The thermodynamics of DNA structural motifs. Annu Rev Bioph Biom 33, 415-440.

[3] Anselmi, C., DeSantis, P. and Scipioni, A. (2005) Nanoscale mechanical and dynamical properties of DNA single molecules. Biophysical Chemistry 113, 209-221.

[4] Dirks, R.M. and Pierce, N.A. (2004) An algorithm for computing nucleic acid base-pairing probabilities including pseudoknots. Journal of Computational Chemistry 25, 1295-1304.

[5] Yin, P., Guo, B., Belmore, C., Palmeri, W., Winfree, E., LaBean, T.H. and Reif, J.H. (2004) In Ferretti, C., Mauri, G. and Zandron, C. (eds.), Tenth International Meeting on DNA Based Computers (DNA10). Springer-Verlag, Milano, Italy, Vol. 3384.

[6] Zhang, M., Sabharwal, C., Tao, W.M., Tarn, T.J., Xi, N. and Li, G.Y. (2004) Interactive DNA sequence and structure design for DNA nano-applications. IEEE Transactions On NanoBioscience 3, 286-292.

[7] Seeman, N.C. (2003) DNA in a material world. Nature 421, 427-431.

[8] Seeman, N.C. (2005) Structural DNA nanotechnology: an overview. Methods Mol Biol 303, 143166.

[9] Lee, S.H. and Mao, C. (2004) DNA nanotechnology. Biotechniques 37, 517-519.

[10] Seeman, N.C. (1982) Nucleic-Acid Junctions and Lattices. J Theor Biol 99, 237-247.

[11] Seeman, N.C. (1995) Molecular Craftwork with DNA. The Chemical Intelligencer 1, 38-47.

[12] Li, X.J., Yang, X.P., Qi, J. and Seeman, N.C. (1996) Antiparallel DNA double crossover molecules as components for nanoconstruction. J Am Chem Soc 118, 6131-6140.

[13] Winfree, E., Liu, F.R., Wenzler, L.A. and Seeman, N.C. (1998) Design and self-assembly of twodimensional DNA crystals. Nature 394, 539-544.

[14] Rothemund, P.W.K., Ekani-Nkodo, A., Papadakis, N., Kumar, A., Fygenson, D.K. and Winfree, E. (2004) Design and characterization of programmable DNA nanotubes. J Am Chem Soc 126, 16344-16352.

[15] Feldkamp, U. and Niemeyer, C.M. (2006) Rational design of DNA nanoarchitectures. Angew Chem Int Ed Engl 45, 1856-1876.

[16] LaBean, T.H., Yan, H., Kopatsch, J., Liu, F.R., Winfree, E., Reif, J.H. and Seeman, N.C. (2000) Construction, analysis, ligation, and self-assembly of DNA triple crossover complexes. $\mathrm{J} \mathrm{Am}$ Chem Soc 122, 1848-1860.

[17] Mao, C.D., LaBean, T.H., Reif, J.H. and Seeman, N.C. (2000) Logical computation using algorithmic self-assembly of DNA triple-crossover molecules. Nature 407, 493-496.

[18] Li, H.Y., Park, S.H., Reif, J.H., LaBean, T.H. and Yan, H. (2004) DNA-templated self-assembly of protein and nanoparticle linear arrays. J Am Chem Soc 126, 418-419.

[19] Liu, D., Park, S.H., Reif, J.H. and LaBean, T.H. (2004) DNA nanotubes self-assembled from triple-crossover tiles as templates for conductive nanowires. P Natl Acad Sci USA 101, 717-722.

[20] Wei, B. and Mi, Y.L. (2005) A new triple crossover triangle (TXT) motif for DNA selfassembly. Biomacromolecules 6, 2528-2532. 
[21] Reishus, D., Shaw, B., Brun, Y., Chelyapov, N. and Adleman, L. (2005) Self-assembly of DNA double-double crossover complexes into high-density, doubly connected, planar structures. $J$ Am Chem Soc 127, 17590-17591.

[22] Yan, H., Park, S.H., Finkelstein, G., Reif, J.H. and LaBean, T.H. (2003) DNA-templated selfassembly of protein arrays and highly conductive nanowires. Science 301, 1882-1884.

[23] He, Y., Tian, Y., Chen, Y., Deng, Z., Ribbe, A.E. and Mao, C. (2005) Sequence symmetry as a tool for designing DNA nanostructures. Angew Chem Int Ed Engl 44, 6694-6696.

[24] Mao, C.D., Sun, W.Q. and Seeman, N.C. (1999) Designed two-dimensional DNA Holliday junction arrays visualized by atomic force microscopy. J Am Chem Soc 121, 5437-5443.

[25] Liu, D., Wang, M., Deng, Z., Walulu, R. and Mao, C. (2004) Tensegrity: construction of rigid DNA triangles with flexible four-arm DNA junctions. J Am Chem Soc 126, 2324-2325.

[26] Ding, B., Sha, R. and Seeman, N.C. (2004) Pseudohexagonal 2D DNA crystals from double crossover cohesion. J Am Chem Soc 126, 10230-10231.

[27] Chelyapov, N., Brun, Y., Gopalkrishnan, M., Reishus, D., Shaw, B. and Adleman, L. (2004) DNA triangles and self-assembled hexagonal tilings. J Am Chem Soc 126, 13924-13925.

[28] Shen, Z.Y., Yan, H., Wang, T. and Seeman, N.C. (2004) Paranemic crossover DNA: A generalized Holliday structure with applications in nanotechnology. J Am Chem Soc 126, 16661674.

[29] Park, S.H., Barish, R., Li, H.Y., Reif, J.H., Finkelstein, G., Yan, H. and LaBean, T.H. (2005) Three-helix bundle DNA tiles self-assemble into 2D lattice or 1D templates for silver nanowires. Nano Lett 5, 693-696.

[30] Mathieu, F., Liao, S.P., Kopatscht, J., Wang, T., Mao, C.D. and Seeman, N.C. (2005) Six-helix bundles designed from DNA. Nano Lett 5, 661-665.

[31] Paukstelis, P.J., Nowakowski, J., Birktoft, J.J. and Seeman, N.C. (2004) Crystal structure of a continuous three-dimensional DNA lattice. Chem Biol 11, 1119-1126.

[32] Chen, J.H. and Seeman, N.C. (1991) Synthesis from DNA of a Molecule with the Connectivity of a Cube. Nature 350, 631-633.

[33] Zhang, Y.W. and Seeman, N.C. (1994) Construction of a DNA-Truncated Octahedron. J Am Chem Soc 116, 1661-1669.

[34] Goodman, R.P., Berry, R.M. and Turberfield, A.J. (2004) The single-step synthesis of a DNA tetrahedron. Chem Commun (Camb) 1372-1373.

[35] Shih, W.M., Quispe, J.D. and Joyce, G.F. (2004) A 1.7-kilobase single-stranded DNA that folds into a nanoscale octahedron. Nature 427, 618-621.

[36] Yan, H., Feng, L.P., LaBean, T.H. and Reif, J.H. (2003) Parallel molecular computations of pairwise exclusive or (XOR) using DNA "String tile" self-assembly. J Am Chem Soc 125, 1424614247.

[37] Rothemund, P.W.K., Papadakis, N. and Winfree, E. (2004) Algorithmic self-assembly of DNA Sierpinski triangles. Plos Biol 2, 2041-2053.

[38] Winfree, E., Yang, X. and Seeman, N.C. (1998) In Landweber, L. and Baum, E. (eds.), 2nd International Meeting on DNA Based Computers (DNA2). DIMACS, Princeton, NJ, Vol. 44, pp. 191-213.

[39] Winfree, E. and Bekbolatov, R. (2004) In Chen, J. and Reif, J. (eds.), 9th International Meeting on DNA Based Computers (DNA9). Springer-Verlag, Madison, Wisconsin, Vol. 2943, pp. 126144. 
[40] Chen, H.L. and Goel, A. (2004) In Ferretti, C., Mauri, G. and Zandron, C. (eds.), 10th Int. Meeting on DNA Based Computers (DNA10). Springer-Verlag, Milano, Italy, Vol. 3384, pp. 274283.

[41] Chen, H.L., Cheng, Q., Goel, A., Huang, M.D. and de Espanes, P.M. (2004), Proc. of the 15th annual ACM-SIAM Symp. on Discrete Algorithms (SODA), pp. 890-899.

[42] Reif, J.H., Sahu, S. and Yin, P. (2006) In Jonoska, N. (ed.). Nanotechnology: Science and Computation ed. Springer-Verlag, pp. 79-104.

[43] Sahu, S. and Reif, J.H. (2006) In Mao, C. and Yokomori, T. (eds.), 12th International Meeting on DNA Computing (DNA 12). Springer-Verlag, Seoul, Korea.

[44] Park, S.H., Pistol, C., Ahn, S.J., Reif, J.H., Lebeck, A.R., Dwyer, C. and LaBean, T.H. (2006) Finite-size, fully addressable DNA tile lattices formed by hierarchical assembly procedures. Angew Chem Int Ed Engl 45, 735-739.

[45] Yan, H., LaBean, T.H., Feng, L.P. and Reif, J.H. (2003) Directed nucleation assembly of DNA tile complexes for barcode-patterned lattices. P Natl Acad Sci USA 100, 8103-8108.

[46] Rothemund, P.W. (2006) Folding DNA to create nanoscale shapes and patterns. Nature 440, 297302.

[47] Liu, Y., Ke, Y. and Yan, H. (2005) Self-assembly of symmetric finite-size DNA nanoarrays. $J$ Am Chem Soc 127, 17140-17141.

[48] Chworos, A., Severcan, I., Koyfman, A.Y., Weinkam, P., Oroudjev, E., Hansma, H.G. and Jaeger, L. (2004) Building programmable jigsaw puzzles with RNA. Science 306, 2068-2072.

[49] Niemeyer, C.M. (2002) The developments of semisynthetic DNA-protein conjugates. Trends Biotechnol 20, 395-401.

[50] Niemeyer, C.M., Sano, T., Smith, C.L. and Cantor, C.R. (1994) Oligonucleotide-directed selfassembly of proteins: semisynthetic DNA--streptavidin hybrid molecules as connectors for the generation of macroscopic arrays and the construction of supramolecular bioconjugates. Nucleic Acids Res 22, 5530-5539.

[51] Niemeyer, C.M., Burger, W. and Peplies, J. (1998) Covalent DNA - Streptavidin conjugates as building blocks for novel biometallic nanostructures. Angew Chem Int Edit 37, 2265-2268.

[52] Park, S.H., Yin, P., Liu, Y., Reif, J.H., LaBean, T.H. and Yan, H. (2005) Programmable DNA self-assemblies for nanoscale organization of ligands and proteins. Nano Lett 5, 729-733.

[53] Cha, J.N., Shimizu, K., Zhou, Y., Christiansen, S.C., Chmelka, B.F., Stucky, G.D. and Morse, D.E. (1999) Silicatein filaments and subunits from a marine sponge direct the polymerization of silica and silicones in vitro. P Natl Acad Sci USA 96, 361-365.

[54] Whaley, S.R., English, D.S., Hu, E.L., Barbara, P.F. and Belcher, A.M. (2000) Selection of peptides with semiconductor binding specificity for directed nanocrystal assembly. Nature 405, 665-668.

[55] Gugliotti, L.A., Feldheim, D.L. and Eaton, B.E. (2004) RNA-mediated metal-metal bond formation in the synthesis of hexagonal palladium nanoparticles. Science 304, 850-852.

[56] Mirkin, C.A., Letsinger, R.L., Mucic, R.C. and Storhoff, J.J. (1996) A DNA-based method for rationally assembling nanoparticles into macroscopic materials. Nature 382, 607-609.

[57] Alivisatos, A.P., Johnsson, K.P., Peng, X., Wilson, T.E., Loweth, C.J., Bruchez, M.P., Jr. and Schultz, P.G. (1996) Organization of 'nanocrystal molecules' using DNA. Nature, 382, 609-611.

[58] Rosi, N.L. and Mirkin, C.A. (2005) Nanostructures in biodiagnostics. Chem Rev 105, 1547-1562. 
[59] Niemeyer, C.M. (2001) Nanoparticles, Proteins, and Nucleic Acids: Biotechnology Meets Materials Science. Angew Chem Int Ed Engl 40, 4128-4158.

[60] Katz, E. and Willner, I. (2004) Integrated nanoparticle-biomolecule hybrid systems: synthesis, properties, and applications. Angew Chem Int Ed Engl 43, 6042-6108.

[61] Seeman, N.C. (1998) Nucleic acid nanostructures and topology. Angew Chem Int Ed Engl 37, 3220-3238.

[62] Le, J.D., Pinto, Y., Seeman, N.C., Musier-Forsyth, K., Taton, T.A. and Kiehl, R.A. (2004) DNAtemplated self-assembly of metallic nanocomponent arrays on a surface. Nano Lett 4, 2343-2347.

[63] Sharma, J., Chhabra, R., Liu, Y., Ke, Y. and Yan, H. (2006) DNA-templated self-assembly of two-dimensional and periodical gold nanoparticle arrays. Angew Chem Int Ed Engl 45, 730-735.

[64] Zhang, J., Liu, Y., Ke, Y. and Yan, H. (2006) Periodic square-like gold nanoparticle arrays templated by self-assembled 2D DNA Nanogrids on a surface. Nano Lett 6, 248-251.

[65] Mitchell, G.P., Mirkin, C.A. and Letsinger, R.L. (1999) Programmed assembly of DNA functionalized quantum dots. J Am Chem Soc 121, 8122-8123.

[66] Parak, W.J., Gerion, D., Zanchet, D., Woerz, A.S., Pellegrino, T., Micheel, C., Williams, S.C., Seitz, M., Bruehl, R.E., Bryant, Z. et al. (2002) Conjugation of DNA to silanized colloidal semiconductor nanocrystalline quantum dots. Chem Mater 14, 2113-2119.

[67] Dujardin, E., Hsin, L.B., Wang, C.R.C. and Mann, S. (2001) DNA-driven self-assembly of gold nanorods. Chem Commun 1264-1265.

[68] Soto, C.M., Srinivasan, A. and Ratna, B.R. (2002) Controlled assembly of mesoscale structures using DNA as molecular bridges. J Am Chem Soc 124, 8508-8509.

[69] Milam, V.T., Hiddessen, A.L., Crocker, J.C., Graves, D.J. and Hammer, D.A. (2003) DNAdriven assembly of bidisperse, micron-sized colloids. Langmuir 19, 10317-10323.

[70] Choi, Y.S., Mecke, A., Orr, B.G., Holl, M.M.B. and Baker, J.R. (2004) DNA-directed synthesis of generation 7 and 5 PAMAM dendrimer nanoclusters. Nano Lett 4, 391-397.

[71] DeMattei, C.R., Huang, B.H. and Tomalia, D.A. (2004) Designed dendrimer syntheses by selfassembly of single-site, ssDNA functionalized dendrons. Nano Lett 4, 771-777.

[72] Keren, K., Krueger, M., Gilad, R., Ben-Yoseph, G., Sivan, U. and Braun, E. (2002) Sequencespecific molecular lithography on single DNA molecules. Science 297, 72-75.

[73] Dai, H.J. (2002) Carbon nanotubes: Synthesis, integration, and properties. Accounts Chem Res 35, 1035-1044.

[74] Banerjee, S., Hemraj-Benny, T. and Wong, S.S. (2005) Covalent surface chemistry of singlewalled carbon nanotubes. Adv Mater 17, 17-29.

[75] Dwyer, C., Guthold, M., Falvo, M., Washburn, S., Superfine, R. and Erie, D. (2002) DNAfunctionalized single-walled carbon nanotubes. Nanotechnology 13, 601-604.

[76] Li, S.N., He, P.G., Dong, J.H., Guo, Z.X. and Dai, L.M. (2005) DNA-directed self-assembling of carbon nanotubes. J Am Chem Soc 127, 14-15.

[77] Williams, K.A., Veenhuizen, P.T.M., de la Torre, B.G., Eritja, R. and Dekker, C. (2002) Nanotechnology - Carbon nanotubes with DNA recognition. Nature 420, 761-761.

[78] Lu, Y.H., Yang, X.Y., Ma, Y.F., Du, F., Liu, Z.F. and Chen, Y.S. (2006) Self-assembled branched nanostructures of single-walled carbon nanotubes with DNA as linkers. Chem Phys Lett 419, 390-393. 
[79] Hazani, M., Hennrich, F., Kappes, M., Naaman, R., Peled, D., Sidorov, V. and Shvarts, D. (2004) DNA-mediated self-assembly of carbon nanotube-based electronic devices. Chem Phys Lett 391, 389-392. 


\section{Figure Captions}

Fig. 1 Schematic drawings of four DNA tiles are shown. Colored lines represent different oligonucleotide strands with arrowheads marking the 3' ends. DAE and DAO are double crossover complexes (as known as DX), TAO is an example of a triple crossover (or TX) tile, and the cross-tile is composed of four arms each of which contains a 4-arm junction.

Fig. 2 AFM images of corrugated (planar) and uncorrugated (tube) versions of cross-tile lattices. The right panel is a $1 \mu \mathrm{m} \times 1 \mu \mathrm{m}$ scan. Adapted with permission from Science, 2003, 301, 1882.

Fig. 3 Schematic drawings of two different triangular tiles (A and C) and AFM images of resulting 2D lattices assembled from triangle tiles (B and D). Adapted with permission from $J$. Am. Chem. Soc. 2004, 126, 2324 (A and B) and J. Am. Chem. Soc. 2004, 126, 10230 (C and D).

Fig. 4 The 3-helix bundle (3HB) DNA tile shown as a schematic trace of the strands through the tile. Different color lines represent different oligonucleotides and arrowheads mark the 3' ends. Six crossover points (paired vertical lines) connect the three double helices (paired horizontal lines) with two crossovers connecting each of the possible pairs of helices. The middle panel is an end view of the $3 \mathrm{HB}$ tile to show the stacking of the helical domains. The right panel is an AFM image of 2D lattice formed from properly programmed $3 \mathrm{HB}$ tiles. Adapted with permission from Nano Lett., 2005, 5, 693-696.

Fig. 5 AFM images of DX tile lattice algorithmically assembled to form fractal Sierpinski triangle patterns. Bright tiles carry an additional stem-loop of DNA projected out of the tile plane which appears taller to the AFM and therefore acts as a topographic marker. The inset to panel A shows a schematic of the target pattern. Panel B is an expanded view of the boxed region in panel A. Panel $\mathrm{C}$ is an expanded view from another section of lattice. Red X's mark tiles with appear to be assembled incorrectly based on visual inspection of the preceding (input) tiles. Scale bars are $100 \mathrm{~nm}$. Adapted with permission from PLoS Biol, 2004, 22041. 
Fig. 6 (A) AFM images of fixed-size DNA tile lattices displaying bound streptavidin molecules (white dots) in arbitrary target patterns. Adapted with permission from Angew. Chem., Int. Ed., 2006, 45, 735-739. (B) Schematic drawing and AFM image of an arbitrary shape formed by folding the single-strand DNA genome of M13 bacteriophage using staple strands (short synthetic oligonucleotides). Adapted with permission from Nature, 2006, 440, 297-302.

Fig. 7 (A) Schematic drawing of cross-tile lattice carrying a biotinylated central strand and streptavidin molecules (blue) binding to the functionalized sites. (B) AFM image showing individual streptavidin proteins at the vertices of the cross tile array. Adapted with permission from Science, 2003, 301, 1882.

Fig. 8 Atomic force microscopy images of the programmed self-assembly of streptavidin on 1D DNA nanotracks. (panels $a$ and $b$ ) AFM image of bare $A * B$ and $A * B *$ nanotrack before streptavidin binding, respectively. Where tiles marked with ' $*$ ' indicate the presence of biotinylated strands. (panels $\mathrm{c}$ and $\mathrm{d}$ ) AFM image of $\mathrm{A} * \mathrm{~B}$ and $\mathrm{A} * \mathrm{~B} *$ nanotrack after binding of streptavidin. All AFM images are $500 \mathrm{~nm}$ x $500 \mathrm{~nm}$. Adapted with permission from Nano Letters, 2005, 5, 729.

Fig. 9 TEM image of gold nanoparticles organized on a self-assembled DX tile lattice using complementary base-pairing interactions. Adapted with permission from Nano Lett., 2004, 4, 2343.

Fig. 10 DNA-mediated deposition of SWNT between two gold electrodes. (A) Schematic representation of hybridization between complementary strands resulting in bridging the two electrodes by the SWNT, and (B) SEM image of two electrode pairs connected by SWNTs 